\title{
Rosmarinic Acid Potently Detoxifies Amylin Amyloid and Ameliorates Diabetic Pathology in a Transgenic Rat Model of Type 2 Diabetes
}

Ling Wu, ${ }^{1,5 \#}$ Paul Velander, ${ }^{1 \#}$ Anne M. Brown, ${ }^{1,2}$ Yao Wang, ${ }^{3}$ Dongmin Liu, ${ }^{2,3}$ David R. Bevan, ${ }^{1,2,4}$ Shijun Zhang, ${ }^{7}$ and $\mathrm{Bin} \mathrm{Xu}^{1,2,4,5,6^{*}}$

${ }^{1}$ Department of Biochemistry, ${ }^{2}$ Center for Drug Discovery, ${ }^{3}$ Department of Human Nutrition, Foods, and Exercise, ${ }^{4}$ School of Neuroscience, Virginia Polytechnic Institute and State University, Blacksburg, Virginia 24061, USA

${ }_{5}^{5}$ Biomanufacturing Research Institute \& Technology Enterprise (BRITE) and Department of Pharmaceutical Sciences, North Carolina Central University, Durham, North Carolina 27707, USA

${ }^{6}$ Affiliated Program Faculty, Duke University Comprehensive Stroke Center, Durham, North Carolina 27710, USA

7 Department of Medicinal Chemistry, Virginia Commonwealth University, Richmond, Virginia 23298, USA

\# Equal contribution

* Correspondence: bxu@nccu.edu or binxu@vt.edu

\section{Table of Contents}

Table S1: Average secondary structure of trimer fragment formation (residues 20 - 29) of amylin with or without the presence of small molecules

Table S2: Average hydrogen bond presence between amylin fragment peptides (20-29), amylin fragment peptides and small molecules, and between small molecules.

Table S3: Average total solvent accessible surface area of amylin fragments (20-29) and small molecules

Figure S1: Cytotoxicity of amylin amyloid on various cell lines and comparison of cytotoxicity of amylin amyloid versus $A \beta$ amyloids. S-3

Figure S2: Control experiments demonstrate that RA has no effects on cell viability....................................................................

Figure S3: Ex vivo efficacies of RA by dot blot analyses...........................

Figure S4: In vivo efficacies of RA by dot blot analyses............................ 
Table S1. Average secondary structure (shown in \%) of trimer fragment formation (residues $20-29)$ of amylin with or without the presence of small molecules. ${ }^{a}$

\begin{tabular}{cccc}
\hline System & Coil & $\beta$-strand & $\alpha$-helix \\
\hline WT & $42 \pm 11$ & $58 \pm 11$ & $0 \pm 0$ \\
RA & $73 \pm 13$ & $28 \pm 13$ & $0 \pm 0$ \\
CA & $63 \pm 17$ & $37 \pm 17$ & $0 \pm 0$ \\
SAA & $52 \pm 17$ & $45 \pm 20$ & $2 \pm 3$ \\
\hline
\end{tabular}

$a$ Percentages represent averages of 3 replicates over the final $100 \mathrm{~ns}$ of simulation time, with associated standard deviations.

Table S2. Average hydrogen bond presence between amylin fragment peptides (20-29), amylin fragment peptides and SMs, and between SMs. ${ }^{a, b}$

\begin{tabular}{cccc}
\hline System & Peptide-Peptide & Peptide-SM & SM-SM \\
\hline WT & $19 \pm 3$ & $\mathrm{n} / \mathrm{a}$ & $\mathrm{n} / \mathrm{a}$ \\
RA & $12 \pm 3$ & $13 \pm 4$ & $3 \pm 1$ \\
CA & $15 \pm 2$ & $4 \pm 4$ & $0.1 \pm 0.1$ \\
SAA & $17 \pm 1$ & $0.7 \pm 0.3$ & $0.1 \pm 0.1$ \\
\hline
\end{tabular}

$\bar{a}$ Averages of 3 replicates over the final $100 \mathrm{~ns}$ of simulation time, with associated standard deviations. SM: Small Molecule.

${ }^{b} \mathrm{n} / \mathrm{a}$ represents not applicable to the system tested.

Table S3. Average total SASA $\left(\mathrm{nm}^{2}\right)$ of amylin fragments (20-29) and SMs. ${ }^{a, b}$

\begin{tabular}{ccc}
\hline System & Peptide & SM \\
\hline WT & $24 \pm 1$ & $\mathrm{n} / \mathrm{a}$ \\
RA & $29 \pm 2$ & $19 \pm 1.6$ \\
CA & $26 \pm 1$ & $14.7 \pm 0.1$ \\
SAA & $26 \pm 1$ & $13.9 \pm 0.1$ \\
\hline
\end{tabular}

${ }^{a}$ Averages of 3 replicates over the final $100 \mathrm{~ns}$ of simulation time, with associated standard deviations.

${ }^{b}$ SASA: solvent accessible surface area. $\mathrm{n} / \mathrm{a}$ represents not applicable to the system tested. 

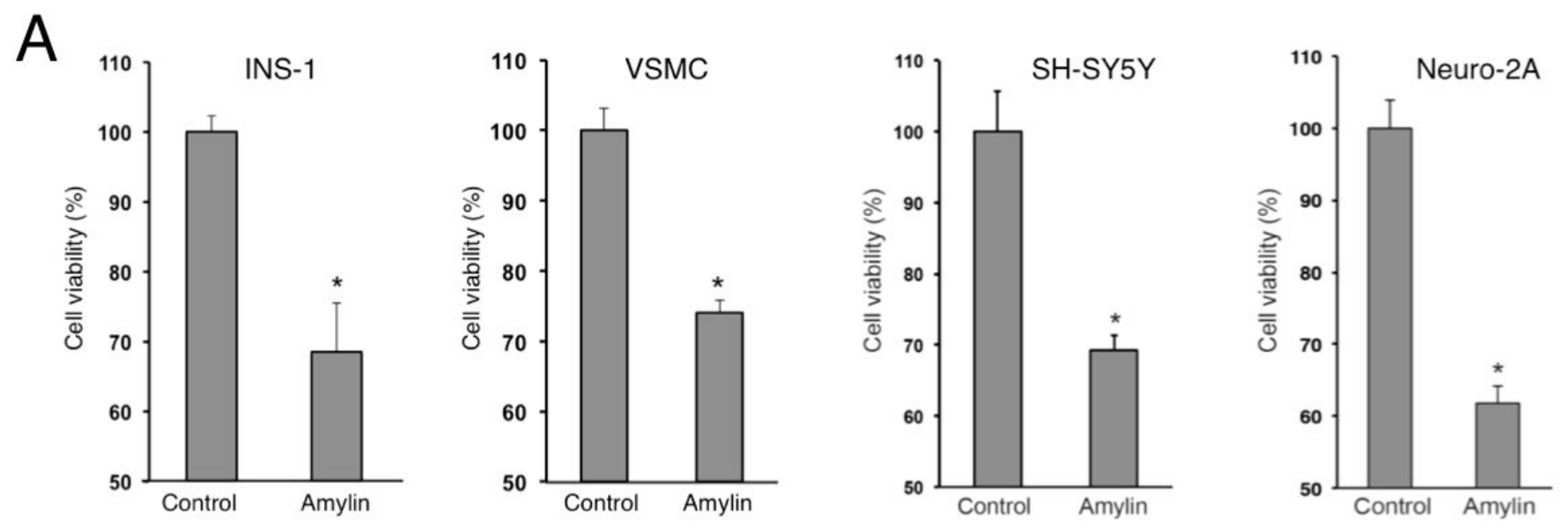

B
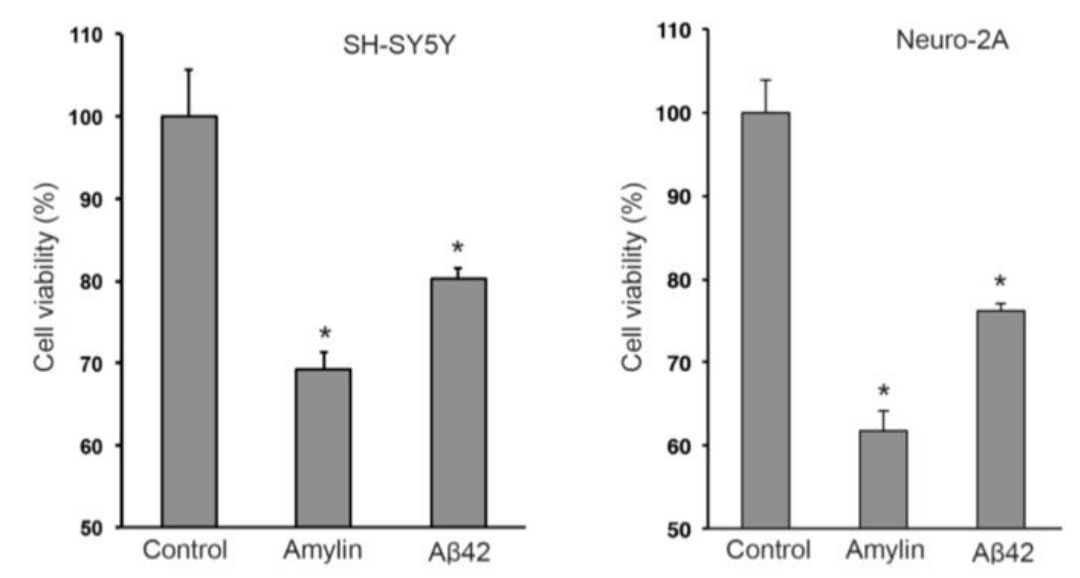

Figure S1. Cytotoxicity of amylin amyloid on various cell lines and comparison of cytotoxicity of amylin amyloid versus $A \beta$ amyloids. (A) Cytotoxicity assays demonstrate the toxic effects of amylin against multiple cell lines relevant to the pancreas, heart, and brain: pancreatic $\beta$ cells INS-1, mouse vascular smooth muscle cells (VSMCs), human SH-SY5Y neuronal cells, and mouse Neuro-2A cells, each at $5 \mu \mathrm{M}$ concentration. (B) Neurotoxicity assays demonstrate that amylin amyloid is more toxic than a well-established control, amyloid $\beta$-peptide $A \beta 42$. Concentrations for each peptide were $10 \mu \mathrm{M}$ for SH-SY5Y treatment or $15 \mu \mathrm{M}$ for Neuro2A treatment. 
A

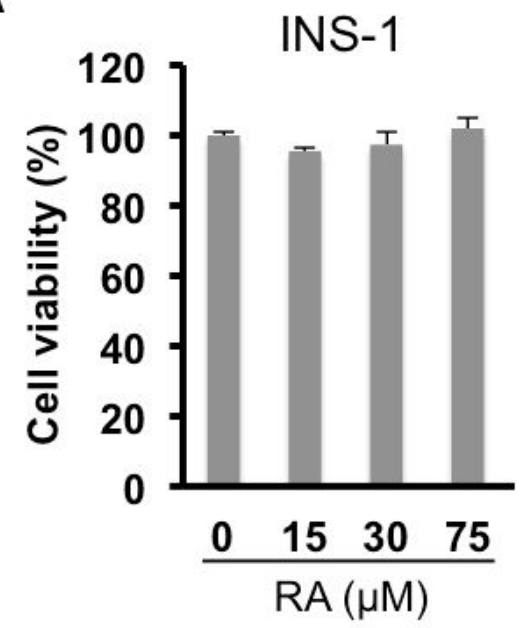

B

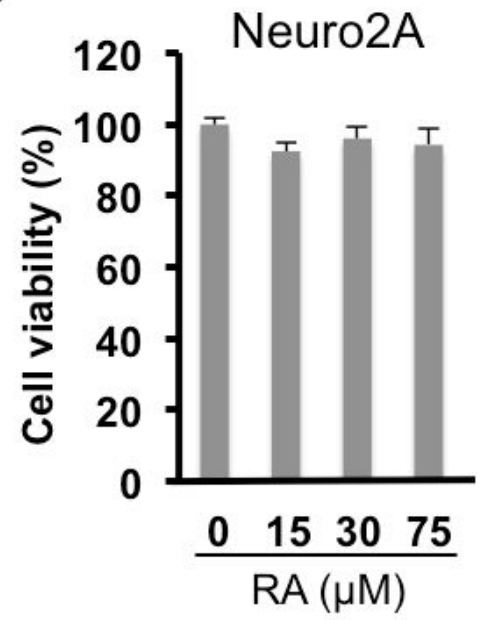

C

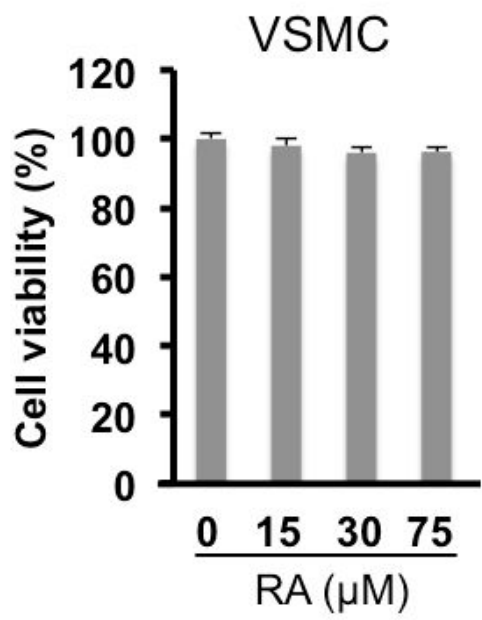

Figure S2. Control experiments demonstrate that RA has no effects on cell viability at the specified concentrations in INS-1 (A), Neuro2A (B), and VSMC cell lines (C). Concentration of amylin was $15 \mu \mathrm{M}$ in each case. 


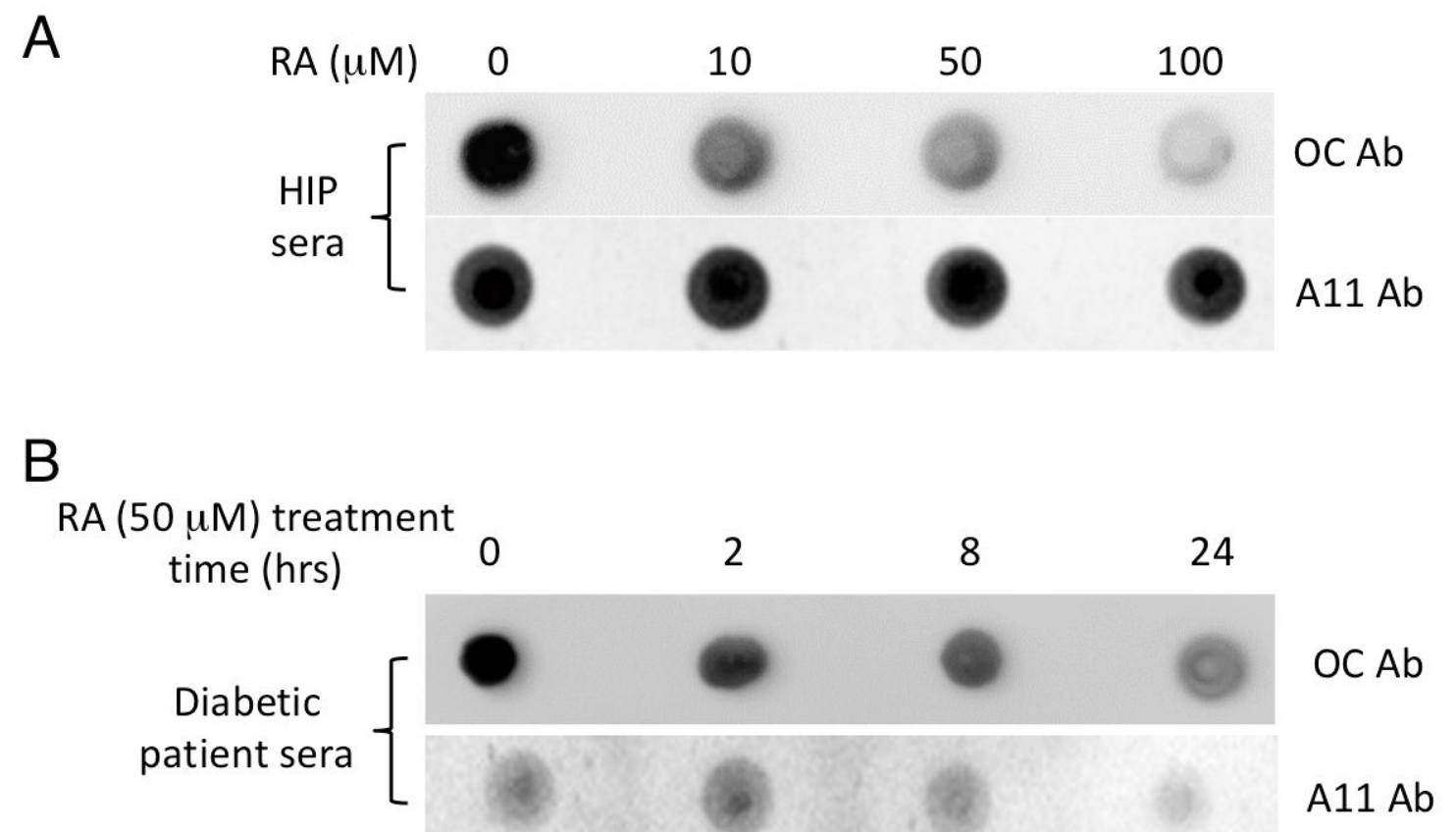

Figure S3. Ex vivo efficacies of RA by dot blot analyses in complement with Western blot analyses in Figure 6. (A) Dot blot analyses of HIP rat sera treated with increased concentrations of RA as indicated. Samples were incubation with RA at indicated concentrations for 3 hours at $37^{\circ} \mathrm{C}$. Equal amount of sera were loaded in each well. Dot blot detections were performed by conformational specific OC antibody (AB2286 from MilliporeSigma; 1:2000 dilution; recognizing amyloid fibrils and fibrillar oligomers, but not prefibrillar oligomers or natively folded proteins) or A11 antibody (AHB0052 from Thermo Fisher Scientific; 1:2000 dilution; recognizing oligomers, but not monomers and fibrils). (B) Dot blot analyses of diabetic patient sera samples treated with $50 \mu \mathrm{M}$ of RA as indicated over a time course of $0,2,8$, and 24 hours. Equal amount of sera were loaded in each well. 


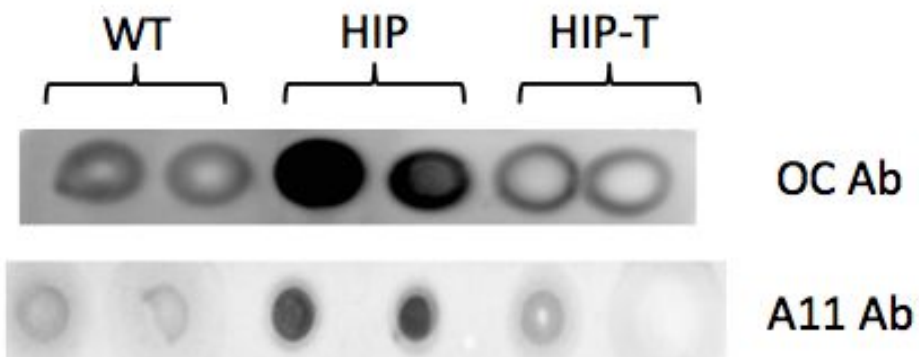

Figure S4. In vivo efficacies of RA by dot blot analyses in complement with Western blot analyses in Figure 7C. Dot blot analyses of serum amylin fibrillar or oligomer levels with conformation specific OC and A11 antibodies. Equal amount of sera were loaded in each well. Comparing with the untreated HIP rat sera, significant reduction of dot blot recognition intensities was observed with RA-treated HIP sera in both OC antibody and A11 antibody detection. Control SD rat sera showed minimal dot blot intensities. 